\title{
Twenty-four-hour rhythmicity of circulating metabolites: effect of body mass and type 2 diabetes
}

\author{
Cheryl M. Isherwood, Daan R. Van der Veen, Jonathan D. Johnston, and Debra J. Skene ${ }^{1}$ \\ Chronobiology Section, Faculty of Health and Medical Sciences, University of Surrey, Guildford, United Kingdom
}

\begin{abstract}
Metabolic profiling of individuals with type 2 diabetes mellitus (T2DM) has previously been limited to single-time-point samples, ignoring time-of-day variation. Here, we tested our hypothesis that body mass and T2DM affect daily rhythmicity and concentrations of circulating metabolites across a 24-h day in 3 age-matched, male groups-lean, overweight/obese (OW/OB), and OW/OB with T2DM-in controlled laboratory conditions, which were not confounded by large meals. By using targeted liquid chromatography/mass spectrometry metabolomics, we quantified 130 plasma metabolites every $2 \mathrm{~h}$ over $24 \mathrm{~h}$, and we show that average metabolite concentrations were significantly altered by increased body mass (90 of 130) and T2DM (56 of 130). Thirty-eight percent of metabolites exhibited daily rhythms in at least 1 study group, and where a metabolite was rhythmic in $>1$ group, its peak time was comparable. The optimal time of day was assessed to provide discriminating biomarkers. This differed between metabolite classes and study groups-for example, phospholipids showed maximal difference at 5:00 AM (lean vs. OW/OB) and at 5:00 PM (OW/OB vs. T2DM). Metabolites that were identified with both robust 24-h rhythms and significant concentration differences between study groups emphasize the importance of controlling the time of day for diagnosis and biomarker discovery, offering a significant improvement over current single sampling.-Isherwood, C. M., Van der Veen, D. R., Johnston, J. D., Skene, D. J. Twenty-four-hour rhythmicity of circulating metabolites: effect of body mass and type 2 diabetes. FASEB J. 31, 5557-5567 (2017). www.fasebj.org
\end{abstract}

KEY WORDS: metabolomics $\cdot$ human $\cdot$ obesity $\cdot$ biological rhythms $\cdot$ circadian clock

It is widely accepted that obesity is the main risk factor for type 2 diabetes mellitus (T2DM) (1). The progression from obesity to T2DM is largely a result of comorbidities, such as systemic inflammation and insulin resistance. Metabolic profiling by using targeted metabolomics, which enables the quantification of more than 100 low-MW intermediates of metabolism, is increasingly used to characterize (pre)diabetic phenotypes and has identified differences in metabolite profiles between those individuals who are obese and those with T2DM (2-5).

ABBREVIATIONS: $\alpha$-AAA, $\alpha$-aminoadipic acid; AC-C18:1, octadecenoylcarnitine; AC-C3, propionylcarnitine; BMI, body mass index; $\mathrm{CV}$, coefficient of variation; FDR, false discovery rate; $\mathrm{HbA1c}$, hemoglobin A1c; LC/MS, liquid chromatography/mass spectrometry; lysoPCa, lysophosphatidylcholine; NEFA, nonesterified fatty acid; OPLS-DA, orthogonal partial least squares discriminant analysis; $\mathrm{OW} / \mathrm{OB}$, overweight/obese; PCaa, diacylphosphatidylcholine; $\mathrm{QC}$, quality control; $\mathrm{SM}$, sphingomyelin; T2DM, type 2 diabetes mellitus; t4-OH-Pro, trans-4-hydroxyproline; TAG, triglyceride

${ }^{1}$ Correspondence: Chronobiology Section, Faculty of Health and Medical Sciences, University of Surrey, GU2 7XH Guildford, United Kingdom. E-mail: d.skene@surrey.ac.uk

This is an Open Access article distributed under the terms of the Creative Commons Attribution 4.0 International (CC BY 4.0) (http:// creativecommons.org/licenses/by/4.0/) which permits unrestricted use, distribution, and reproduction in any medium, provided the original work is properly cited.

doi: 10.1096/fj.201700323R

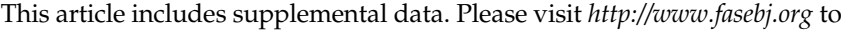
obtain this information.
Recent work by our group and others has shown a 24-h variation in the human metabolome in healthy individuals, analyzed by using a range of analytical platforms (6-12), which has demonstrated that an estimated $15-20 \%$ of the metabolome is rhythmic in blood $(6,7)$. Transgenic mice that carry targeted genetic manipulation of circadian clock genes also exhibit a phenotype that involves defective metabolism, and associations between the circadian timing system and metabolic responses have been reported in humans (13). Reviews of these studies, including the higher incidence of obesity, T2DM, and related disorders in shift workers, have recently been published $(14,15)$.

Existing metabolomics studies in T2DM have been restricted to the analysis of single-time-point, mostly fasting, samples, which cannot characterize the effect of increased body mass and T2DM on rhythmic metabolites. Characterizing 24-h metabolite rhythms in T2DM compared with age- and body mass-matched controls may therefore provide novel insights into the etiology and progression of T2DM. Identification of the optimal time of day for blood sampling - when metabolite levels show the biggest difference between T2DM and controls-would also provide more discriminating diagnostic biomarkers, rather than taking a single morning fasting sample.

We thus assessed the effect of increased body mass [overweight/obese (OW/OB)] and T2DM on 24-h rhythms of circulating metabolites in men by using a quantitative 
targeted liquid chromatography/mass spectrometry (LC/MS) metabolomics approach. As T2DM is often accompanied by obesity, we set out to distinguish the effects of T2DM from those of increased body mass by incorporating both a lean and an OW/OB control group into the current study design.

\section{MATERIALS AND METHODS}

All aspects of the study were conducted in accordance with the Declaration of Helsinki and conformed to international ethical standards. A favorable ethical opinion was obtained from the Surrey Research Ethics Committee and the University of Surrey Ethics Committee. Written, informed consent was obtained from all participants.

\section{Prelaboratory session}

The screening process and study protocol have been described previously $(16,17)$. In brief, initial screening was conducted via a self-reported medical history questionnaire, and was confirmed by the participant's general practitioner. Participants who were receiving medication that was known to influence the endogenous circadian timing system or who had a history of hypoglycemic episodes or hemoglobin $\mathrm{A} 1 \mathrm{c}(\mathrm{HbA} 1 \mathrm{c})>8.5 \%(69 \mathrm{mM})$ were excluded. Additional clinical screening was performed to assess general health, sleep patterns, and diurnal preference (morningness/eveningness).

Effects of body mass index (BMI) and T2DM were assessed separately within the study by using 3 study groups of men: 1) lean, 2) OW/OB, and 3) T2DM. Participants in the lean and OW/ $\mathrm{OB}$ groups were matched for age and glucose homeostasis markers (HbA1c, fasting glucose and insulin), whereas participants in the OW/OB and T2DM groups were matched for age and BMI. Participant $(n=23)$ demographics for metabolomics analyses are shown in Table $\mathbf{1}$.

To minimize sleep debt and any effects of nutritional status or exercise, a strict prestudy protocol was prescribed the week before study entry at the Surrey Clinical Research Centre. Participants' in-bed time was aligned to the sleep schedule of the laboratory session (10:30-6:30 AM), without deviation by more than $15 \mathrm{~min}$. Within $90 \mathrm{~min}$ of waking, participants were asked to obtain at least $15 \mathrm{~min}$ of natural outdoor light to maximize circadian entrainment. Excessive exercise was also avoided. Compliance was verified by using continuously worn rest-activity monitors (Actiwatch-L; Cambridge Neurotechnology, Cambridge, United Kingdom). Alcohol and caffeine consumption was prohibited throughout the prestudy and laboratory session. For the first $4 \mathrm{~d}$ of the prestudy protocol, participants had to consume their meals within set time windows (breakfast 7:009:00 AM, lunch 12:00-2:00 PM, and dinner 5:30-7:30 PM) (16). During the last $3 \mathrm{~d}$ of the prestudy protocol, participants could only consume food that had been provided by the research team during these same time windows. Participants were given the same breakfast but could choose their other meals from a set selection of ready meals with similar macronutrient content. Total daily food energy ( $~ 35 \%$ derived from fat) was $150 \%$ of the basal metabolic rate using the Schofield equation (18).

\section{Laboratory session}

During the laboratory session, light intensity, physical activity, and meals were strictly controlled, as illustrated in Fig. 1. Participants lay semirecumbent throughout. During lights on (6:30 AM-10:30 PM), light intensity was $\sim 600$ lux in the direction of gaze. During the light period, small meals, which were composed of isocaloric Fortisip (Nutricia, Schiphol, The Netherlands) milkshakes, were given hourly from 3:30 PM (d 1 ), and water was available ad libitum. Individual food energy intake was set at $110 \%$ of the basal metabolic rate using the Schofield equation (18). During lights off (10:30 PM to 6:30 AM, 0 lux), participants had the opportunity to sleep.

Blood samples ( $n=13$ per participant) were drawn into anticoagulant (dipotassium-EDTA) tubes every $2 \mathrm{~h}$ from 7:00 AM on $\mathrm{d} 1$ for $24 \mathrm{~h}$, spun $\left(3000 \mathrm{~g}, 10 \mathrm{~min}, 4^{\circ} \mathrm{C}\right)$ to isolate plasma, and stored at $-80^{\circ} \mathrm{C}$ until analysis.

\section{Analysis of plasma metabolites}

Samples for glucose and triglyceride (TAG) analyses were thawed overnight at $4^{\circ} \mathrm{C}$ before undergoing measurement in the ILab 650 Clinical Analyzer (Instrumentation Laboratory, North Risley, United Kingdom). The combined flow injection analysis and LC/MS targeted metabolomics analysis was performed by using the AbsoluteIDQ p180 kit (Biocrates Life Sciences AG, Innsbruck, Austria) and a Waters Xevo TQ-S mass spectrometer that was coupled to an Acquity UPLC system (Waters, Milford, MA, USA). Plasma samples (10 $\mu \mathrm{l})$ were processed on four 96well plates-each plate containing all time points from at least 1 participant from each group - and were measured within 1 mo of each other. The samples on each plate were randomly assigned to minimize any effect of assay drift.

Human plasma-based quality controls (QCs) with analytes added in 3 defined concentrations-low (QC1), medium (QC2), and high (QC3) - were provided with the kit. Medium quality control (QC2), repeated every 20 samples on each assay plate, was used to calculate the intra- and interplate coefficient of variation $(\mathrm{CV} \%)$. Data were normalized between batches by using the results of QC2 repeats across the plate $(n=4)$ and between

TABLE 1. Participant screening data of lean, $O W / O B$, and T2DM study groups

\begin{tabular}{lccc}
\hline \hline Variable & Lean, $n=8$ & OW/OB, $n=9$ & T2DM, $n=6$ \\
\hline Age $(\mathrm{yr})$ & $53.6 \pm 6.0$ & $51.0 \pm 7.7$ & $57.3 \pm 4.8$ \\
BMI $\left(\mathrm{kg} / \mathrm{m}^{2}\right)$ & $23.2 \pm 1.4^{* * *}$ & $29.8 \pm 2.3^{\# \# \#}$ & $32.2 \pm 2.5$ \\
Waist circumference $(\mathrm{cm})$ & $88.9 \pm 6.5^{* * *}$ & $105.1 \pm 3.9^{\# \# \#}$ & $112.8 \pm 8.9$ \\
Glucose $(\mathrm{mM})$ & $4.2 \pm 0.7^{* * *}$ & $4.9 \pm 0.7^{* *}$ & $6.7 \pm 1.6$ \\
Insulin (pM) & $28.1 \pm 16.8^{* *}$ & $39.9 \pm 18.5^{* *}$ & $110.5 \pm 85.3$ \\
HbAlc (\%) & $5.4 \pm 0.4^{* * *}$ & $5.3 \pm 0.5^{* * *}$ & $6.9 \pm 0.9$ \\
BP systolic (mmHg) & $129.4 \pm 9.7^{*}$ & $133.8 \pm 11.1$ & $146.8 \pm 10.6$ \\
BP diastolic (mmHg) & $84.0 \pm 10.2$ & $85.1 \pm 10.7$ & $88.8 \pm 9.0$ \\
\hline
\end{tabular}

Data are given as means \pm SD. BP, blood pressure. $* P<0.05, * * P<0.01, * * * P<0.001$ vs. T2DM;

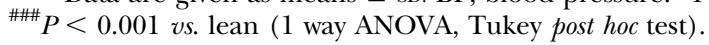




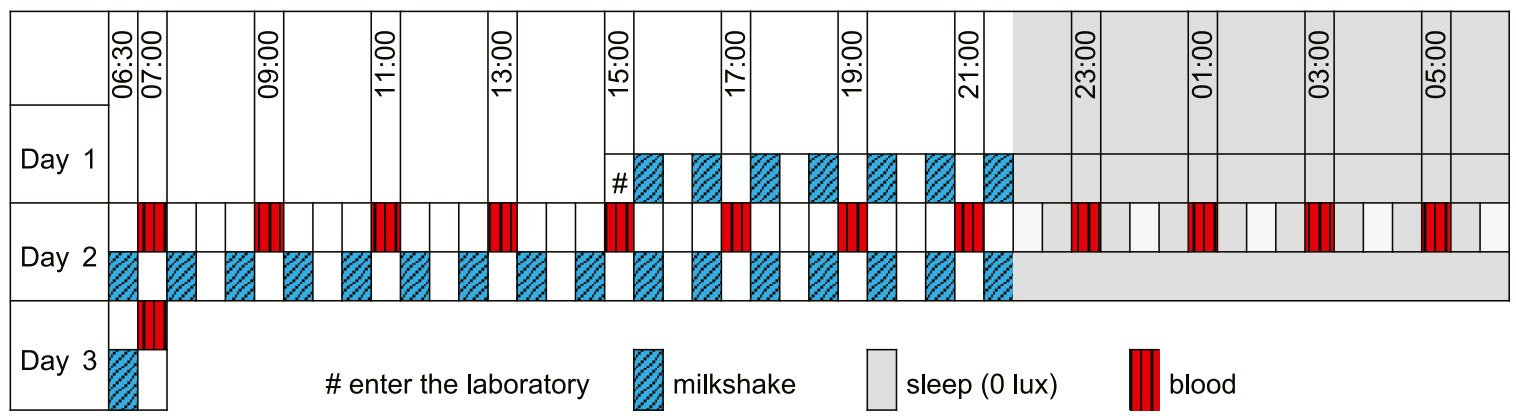

Figure 1. Study protocol. Participants $(n=23)$ entered the clinical laboratory at 3:00 PM on d 1 and lay semirecumbent throughout. The next day (d 2), during the light period from 6:30 AM to 10:30 PM ( 600 lux, in direction of gaze), participants were given hourly isocaloric milkshakes that commenced at 6:30 AM and water was available ad libitum. During the dark period (10:30 PM to 6:30 AM, 0 lux), they had the opportunity to sleep. Blood was drawn every 2 h from 7:00 AM on d 2 until 7:00 AM the next day (d 3).

plates $(n=4)$ using METIDQ software (QC2 correction; Biocrates Life Sciences AG). Metabolites were excluded if the CV\% of QC2 was $>30 \%$ or if all 3 groups contained $>25 \%$ of samples that were below the limit of detection, below the lower limit of quantification, or above the limit of quantification or blank out of range ( $n=54$ excluded) (19). Intraplate $C V \%$ of the remaining 130 quantified metabolites was $4.8 \pm 0.3 \%$ (mean \pm SEM) and interplate CV\% was $10.2 \pm 0.5 \%$ (mean \pm SEM). Quantified metabolites were composed of 20 amino acids and 9 biogenic amines measured by LC/MS (7-point calibration using isotope-labeled internal standards) and 10 acylcarnitines, 13 lysophosphatidylcholines (lysoPCas), 30 diacylphosphatidylcholines (PCaas), 34 acyl-alkyl-phosphatidylcholines, 9 sphingomyelins (SMs), and 5 hydroxysphingomyelins measured by flow injection analysis (1-point calibration using isotope-labeled internal standards for acylcarnitines or unlabeled internal standards for lipids). Lipids were quantified by using nonphysiologic lipids as internal standards, 2 for PCs, 1 for lysoPCs, and 1 for sphingolipids. As a result of the unavailability of isotope-labeled lipid standards, quantification, but not precise identification, was possible (lipid signals measured with the AbsoluteIDQp180 kit having a number of possible isobars and/or isomers).

\section{Data analysis}

To assess 24-h metabolite rhythmicity, we performed cosinor analysis on mean $z$-score values- $z$ score calculated across $24 \mathrm{~h}$ per individual (12) - for all participants. Peak time (acrophase), amplitude, and significance of a cosine fit $(P<0.05)$ were determined in each case (Matlab 2013b; MathWorks, Natick, MA, USA). Principal component analysis and orthogonal partial least squares discriminant analysis (OPLS-DA; SIMCA-P v.13.0 software; Umetrics, Malmo, Sweden) models (lean vs. OW/OB and $\mathrm{OW} / \mathrm{OB}$ vs. T2DM) were used to identify metabolites with the most variance between study groups. Validation of the OPLS-DA models was provided by permutation analysis (999 permutations) and a CV ANOVA $P$ value of the model. Differences in individual metabolite levels were analyzed in $\mathrm{R}$ (version 3.2.2; The R Foundation, Vienna, Austria) using the aov function in the statistics package (19). Linear models were fitted to the study group and time of day (13 time points, repeated measures per individual), with individual participants as covariates. Significant differences between study group, time of day, and their interaction were determined by using 2-way ANOVA. Significance values were corrected for multiple comparisons according to the Benjamini-Hochberg false discovery rate (FDR). Differences between metabolites were considered significant with an FDR $<0.05$. Missing values $(n=16 ; 5.4 \%)$ were left blank and were not taken into account for both the univariate and multivariate analyses. Missing values were not clustered in a particular sampling time (no time point had $>4$ missing samples) or study group $(n=7,5$, and 4 in lean, OW/OB, and T2DM groups, respectively). The time point at which most metabolites within each class had the maximum concentration difference between lean and $\mathrm{OW} / \mathrm{OB}$ groups and between $\mathrm{OW} / \mathrm{OB}$ and T2DM groups was calculated by using absolute values and illustrated in polar plots by using $\mathrm{R}$. We performed quantitative enrichment analysis (lean $v s$. OW/OB and OW/OB vs. T2DM) by using MetaboAnalyst 3.0 (www.metaboanalyst.ca) (20).

\section{RESULTS}

To assess the effect of increased body mass, we compared the OW/OB group with the lean group, both of which were matched for glucose homeostasis. Comparison of the $\mathrm{OW} / \mathrm{OB}$ and T2DM groups enabled us to compare the effect of T2DM per se in weight-matched groups. Table 1 presents the screening demographic data for the 3 study groups. The T2DM group had significantly higher glucose, insulin, and $\mathrm{HbA1c}$ than both the $\mathrm{OW} / \mathrm{OB}$ and lean groups (Table 1).

Assessment of daily rhythmicity, as determined by cosinor analysis of 24-h metabolite profiles, identified $50(38 \%)$ of 130 metabolites with significant daily rhythms $(P<0.05)$ in at least one of the study groups (Fig. 2$), 84 \%$ of which peaked during lights on. Fourteen $(28 \%)$ of these 50 metabolites displayed 24 -h daily rhythms in all 3 groups, namely, citrulline, proline, sarcosine, symmetrical dimethylarginine, acetylcarnitine, AC-C3 (propionylcarnitine), butyrylcarnitine, hexadecanoylcarnitine, $4 \times$ lysoPCa (C18:1, C18:2, C20:3, and C20:4), and 2× PCaa (C32:1 and C36:5). The 24-h profiles of these rhythmic metabolites are shown in Fig. 3. An additional $11(22 \%)$ of 50 metabolites, namely, alanine, glycine, isoleucine, tyrosine, valine, $\alpha$-aminoadipic acid ( $\alpha$-AAA), AC-C18: 1 (octadecenoylcarnitine), PCaa (C36:1, C36:3, C40:2), and acyl-alkyl-phosphatidylcholine (C36:2) displayed daily rhythms in the lean and OW/OB groups, but not in the T2DM group, whereas $5(10 \%)$ of 50 metabolites displayed daily rhythms in the OW/OB and T2DM groups, but not in the lean group, namely, tetradecenoylcarnitine and octadecanoylcarnitine, $2 \times 1$ lysoPCa (C16:0 and C18:0), and PCaa (C36:2). Also shown in Fig. $2,20(40 \%)$ of 50 metabolites displayed a daily rhythm in only 1 study group. Where a metabolite was rhythmic 


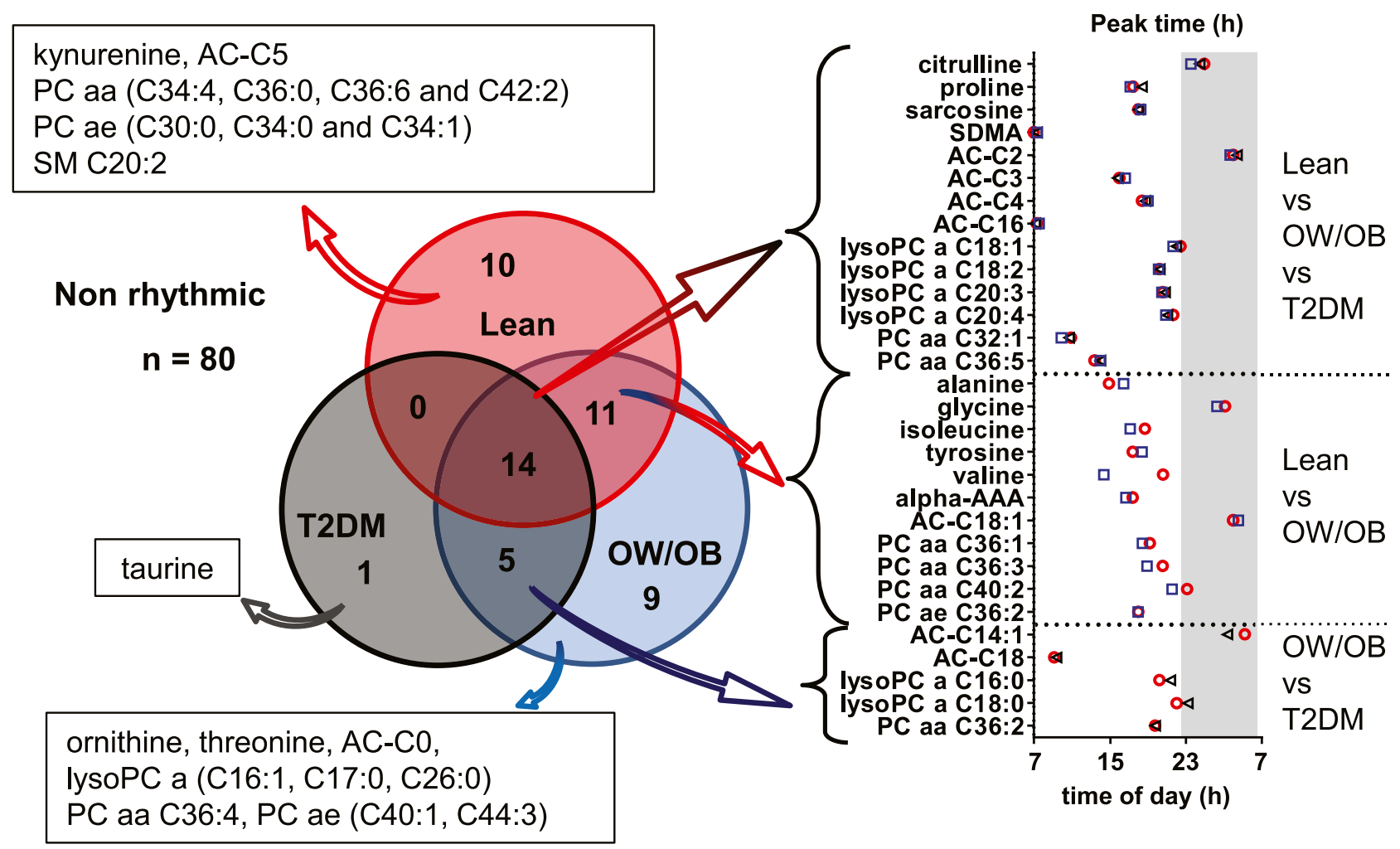

Figure 2. Metabolites with a significant cosine rhythm in the 3 study groups. A) Venn diagram showing the metabolites (name and number) with a significant cosine rhythm in the lean, OW/OB, and T2DM groups. B) Peak times of the metabolites with a significant cosine rhythm in more than 1 study group is also displayed. Peak time of day for each metabolite in the lean (red circle), OW/OB (blue square), and T2DM (black triangle) groups is shown. The dark condition (10:30 PM to 6:30 AM, 0 lux) is illustrated on the graph as a gray-shaded area, and time of day (hour) is displayed on the $x$ axis.

in $>1$ group $[n=30(60 \%)$ of 50$]$, its peak time was comparable between groups (within $2 \mathrm{~h}$ ), with the exception of valine (6-h difference). Peak times of these metabolite rhythms are illustrated in Fig. 2 and are further detailed (peak time and amplitude) in Supplemental Table 1. There were no significant differences in the amplitude of metabolite rhythms between study groups. In addition to the panel of metabolites measured, plasma glucose concentrations displayed a significant cosine rhythm in the lean group only $(P<0.05)$, with a peak time of $15.02 \mathrm{~h}$, and TAG displayed significant 24-h rhythms in the OW/OB and T2DM groups $(P<0.05)$, with peak times of 15.80 and $14.13 \mathrm{~h}$, respectively.

A principal component analysis of all samples-130 metabolites, 13 time points, and 285 observations-was performed $\left[R^{2} X\right.$ (cumulative), 0.877; $Q^{2}$ (cumulative), $0.736 ; n=16$ components]. The OPLS-DA score plot of the lean and OW/OB groups in Fig. $4 A$ demonstrates a clear separation of the 2 groups $\left[R^{2} X\right.$ (cumulative), $0.636 ; R^{2} Y$ (cumulative), 0.913; $Q^{2}$ (cumulative), $0.886 ; n=5$ components, validated by permutation analysis; intercepts: $R^{2}=0.0,0.187$; and $Q^{2}=0.0,-0.435$; and CV ANOVA $P$ value lean $v s$. OW/OB, $\left.P=2.12^{-85}\right]$. Variance within the model that was revealed in the loading plot (Fig. $4 B$ ) illustrates that the amino acids, biogenic amines, and acylcarnitines were mainly negatively correlated, whereas the phospholipids were mainly positively correlated within the model. The OPLS-DA score plot of the OW/OB and T2DM groups also demonstrates a clear separation of the groups $\left[R^{2} X\right.$ (cumulative), $0.658 ; R^{2} Y$ (cumulative), 0.913; $Q^{2}$ (cumulative), 0.879; $n=5$ components, validated by permutation analysis; intercepts: $R^{2}=0.0,0.202$; and $Q^{2}=0.0,-0.478$; and CV ANOVA $P$ value OW/OB vs. T2DM, $P=7.60^{-75}$; Fig. $4 C]$. In contrast, there was no obvious pattern in the variance between the $\mathrm{OW} / \mathrm{OB}$ and T2DM groups according to metabolite class in the loadings plot (Fig. 4D). $P$ (loading) values of all of the metabolites in both OPLS-DA models are shown in Supplemental Table 2.

We performed 2-way ANOVA (study group and time of day as factors) to assess the effect of increased body mass and T2DM on metabolite 24-h concentrations. Mean \pm SEM concentrations (micromolar) of the metabolites measured in the lean, $\mathrm{OW} / \mathrm{OB}$, and T2DM groups and of the metabolites with significant group differences (FDR < 0.05) between lean and OW/OB groups and OW/OB and T2DM groups are shown in Supplemental Table 3. Ninety metabolites $(69 \%)$ were significantly different between the lean and OW/OB groups (FDR $<0.05), 70(78 \%)$ of 90 had significantly lower concentrations in the OW/OB group, comprising mainly phospholipids [66 (94\%) of 70], which confirmed the patterns that were observed in the OPLS-DA (Fig. 4). 

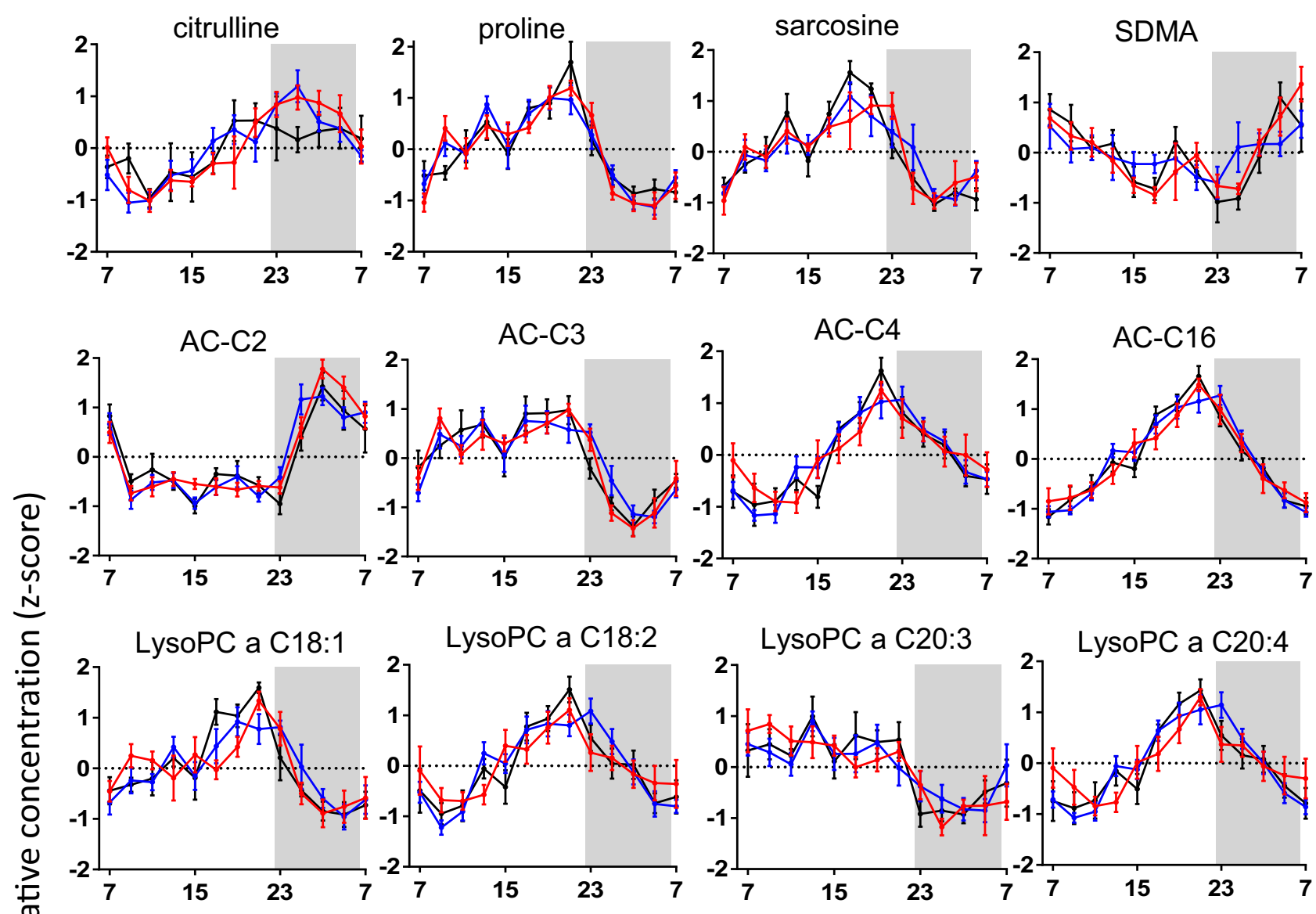

\section{$\mathrm{PC}$ aа C32:1}

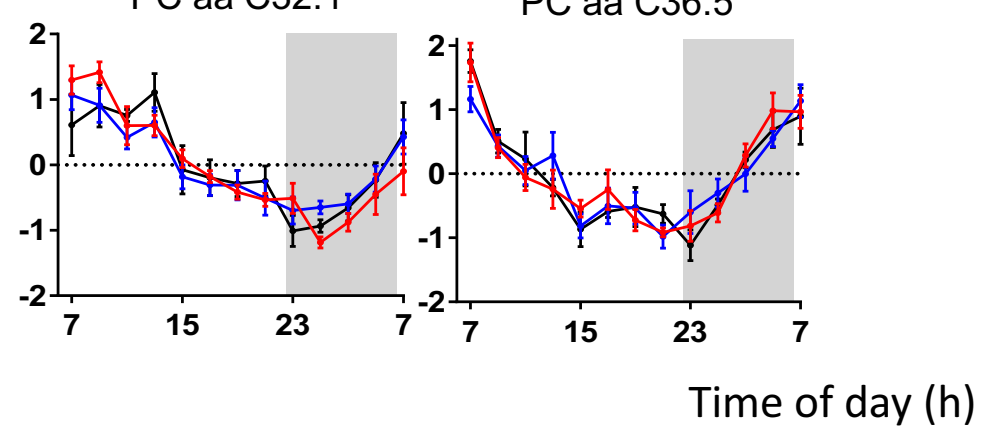

Figure 3. Metabolites with daily 24-h rhythms in all study groups. Metabolites with significant cosinor rhythms $(n=14)$ in the lean (red), OW/OB (blue), and T2DM (black) groups peak at a similar time of day, with no significant difference in relative amplitude. Relative concentration ( $z$ score) is displayed on the $y$ axis, values are mean \pm sEM. The dark condition (22:30-06:30 h, 0 lux) is illustrated on the graph as a gray-shaded area, and time of day (h) is displayed on the $x$ axis.

Quantitative enrichment analysis indicated that tyrosine metabolism (3-fold enrichment, $P=0.075$ ), catecholamine biosynthesis (3-fold enrichment, $P=0.075$ ), and phenylalanine and tyrosine metabolism (2-fold enrichment, $P=0.120$ ) were the top 3 pathway-associated metabolite sets related to increased body mass. None of these pathways, however, was significantly enriched $(P>0.05)$.

When comparing T2DM and OW/OB groups, 56 (43\%) of 130 metabolites demonstrated significant differences in concentration between the 2 groups (Supplemental Table 3). More than $60 \%$ of these metabolites [34 (61\%) of 56] had significantly higher concentrations in T2DM, namely, amino acids (alanine, phenylalanine, glutamate, proline, and tyrosine), biogenic amines $[\alpha$-AAA, sarcosine, trans-4-hydroxyproline $(\mathrm{t} 4-\mathrm{OH}-$ Pro)], acylcarnitines (AC-C0, AC-C3), 22× PC, and 2 sphingolipids (SM C20:2 and SM C26:1). Metabolites with significantly lower concentrations in T2DM [22 (39\%) of 56] were glutamine, histidine, ornithine, serine, AC-C18 (octadecanoylcarnitine), AC-C18:1, octadecadienylcarnitine, $5 \times$ lysoPC, $8 \times \mathrm{PC}$, and 2 sphingolipids [SM C16:0 and $\mathrm{SM}(\mathrm{OH}) \mathrm{C} 16: 1]$. The 24-h profiles of the metabolites with significant concentration differences between the T2DM group and OW/OB controls are shown in Fig. 5. The top 4 metabolite pathways that were associated with T2DM (between the OW/OB and T2DM study groups) were histidine metabolism 
Figure 4. OPLS-DA plots separated according to study group. A) Score plot of lean (red) vs. $\mathrm{OW} / \mathrm{OB}$ (blue) groups; $R^{2} X$ (cumulative), $0.636 ; R^{2} Y$ (cumulative), 0.913; and $Q^{2}$ (cumulative), 0.886 (validated by permutation analysis). B) Score plot of OW/OB (blue) vs. T2DM (black) groups; $R^{2} X$ (cumulative), $0.658 ; R^{2} Y$ (cumulative), 0.913 ; and $Q^{2}$ (cumulative), 0.879 (validated by permutation analysis). C) Loading plot of lean $v s$. OW/OB groups where negative $P$ (loading) values represent metabolites with higher concentrations, and positive $P$ (loading) values represent metabolites with lower concentrations in $\mathrm{OW} / \mathrm{OB}$ compared with the lean group. Every fifth metabolite is labeled on the $x$ axis. $D)$ Loading plot of $\mathrm{OW} / \mathrm{OB}$ vs. T2DM groups where negative $P$ (loading) values represent metabolites with higher concentrations, and positive $P$ (loading) values represent metabolites with lower concentrations in T2DM compared with the OW/OB group. Only metabolites whose error bars did not span the $x$ axis have been plotted. Every second metabolite is labeled on the $x$ axis. Metabolites are colored according to class; amino acids and biogenic amines (blue), acylcarnitines (green), PCaa (yellow), acyl-alkyl-phosphatidylcholine (PCae; light orange), lysoPC (dark orange), and sphingomyelin (brown). For a full list of all metabolites and their corresponding $P$ (loading) values, see Supplemental Table 2.

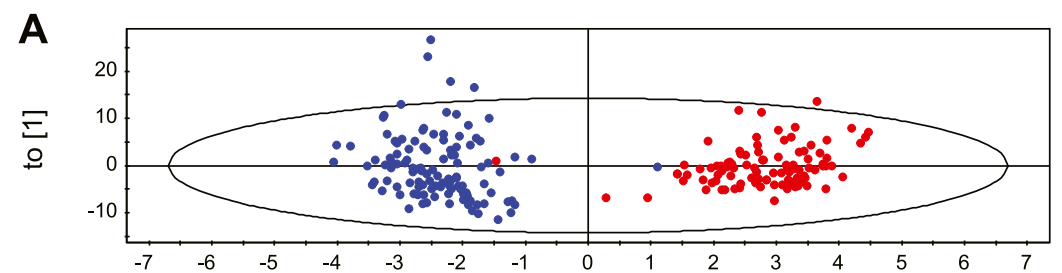

B

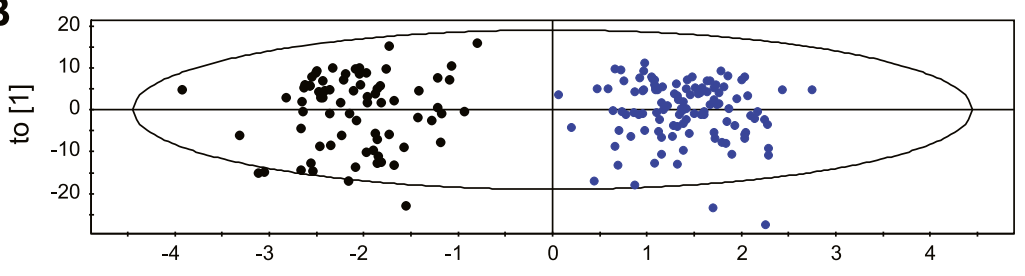

C 0.3 to [1]

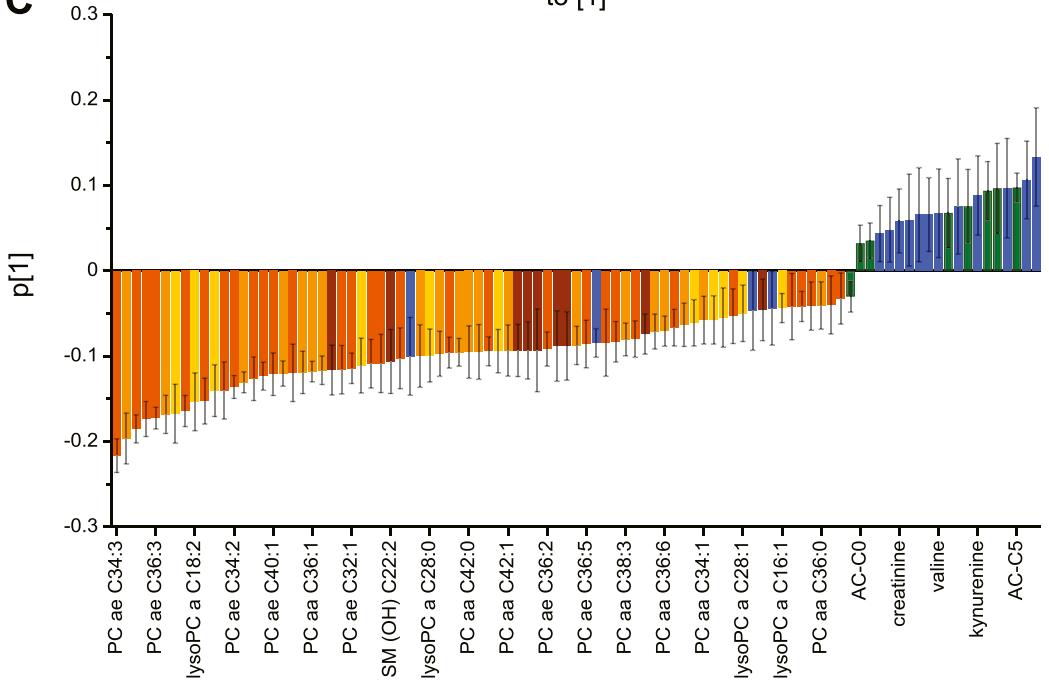

D

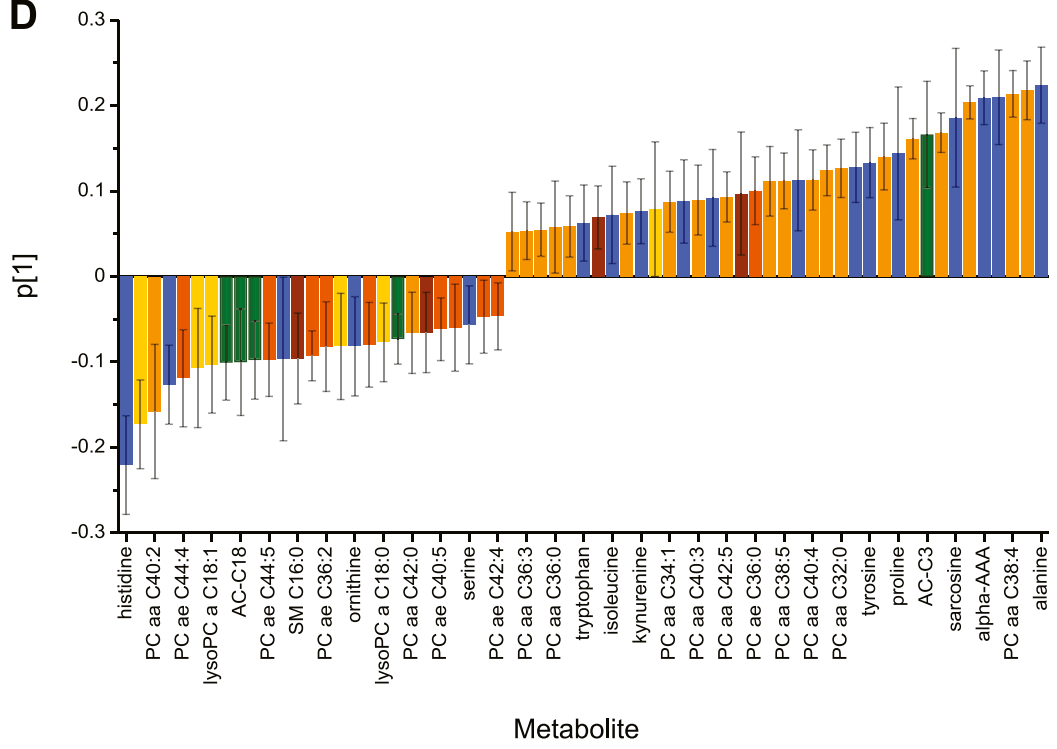

(4-fold enrichment, $P=0.036$ ), pyrimidine metabolism (2.5-fold enrichment, $P=0.123$ ), purine metabolism (2.5fold enrichment, $P=0.123$ ), and glutamate metabolism (2.5-fold enrichment, $P=0.123$ ). After FDR correction, the histidine metabolism pathway was not significantly enriched.

Figure 5 also shows the 24-h profiles of glucose and TAG. The 24-h average glucose levels were significantly higher $(P<0.001)$ in the T2DM group (mean \pm SEM, $7.74 \pm$ $0.32 \mathrm{mM})$ compared with the OW/OB group $(6.53 \pm 0.15$ $\mathrm{mM})$ and lean controls $(6.08 \pm 0.6 \mathrm{mM})$. The 24 -h average TAG concentrations were significantly lower $(P<0.01)$ in the T2DM group $(1.43 \pm 0.05 \mathrm{mM})$ compared with the $\mathrm{OW} / \mathrm{OB}$ group $(1.72 \pm 0.23 \mathrm{mM})$, and were significantly higher $(P<0.001)$ in the OW/OB group compared with lean controls $(1.15 \pm 0.04 \mathrm{mM})$. 


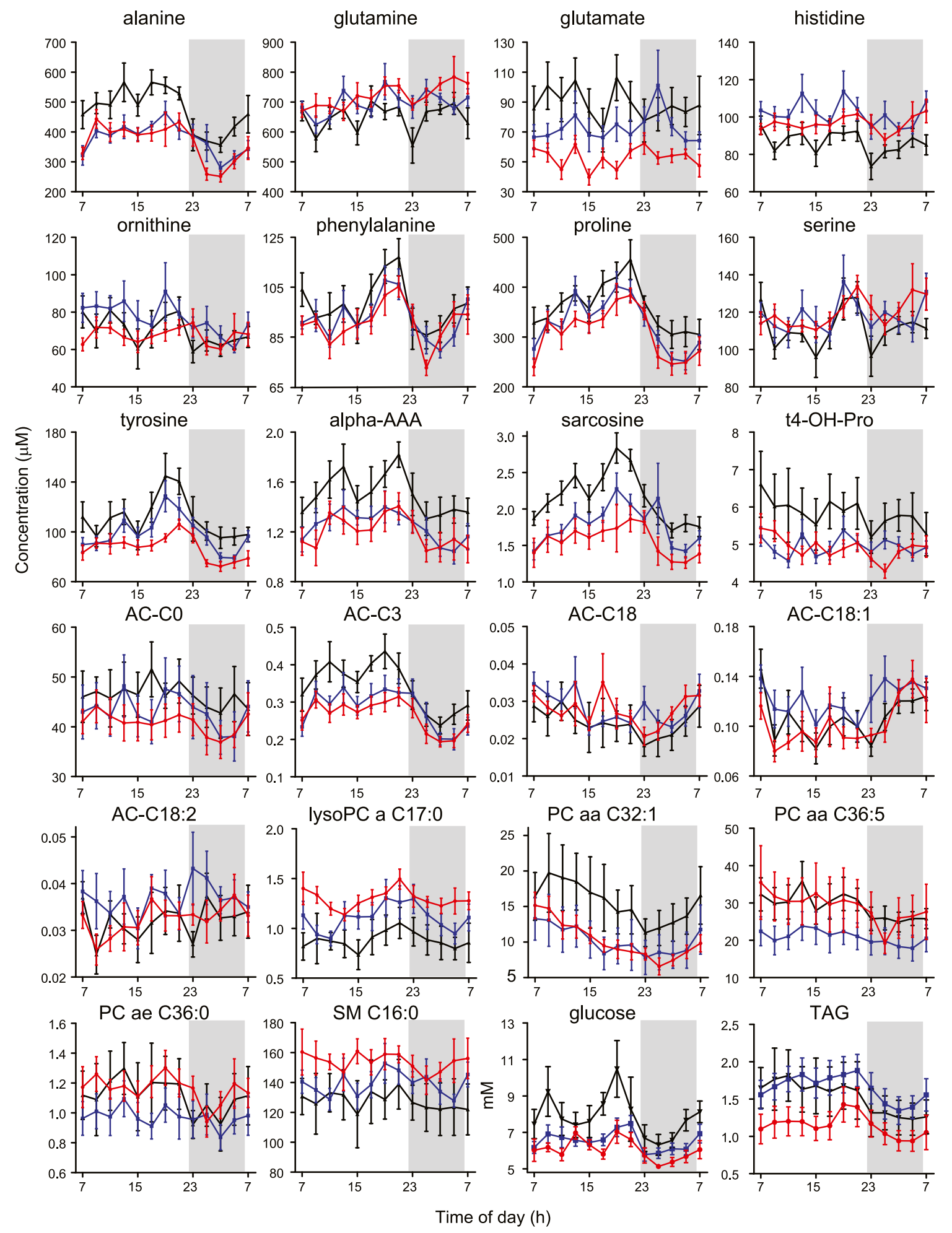

Figure 5. Twenty-four-hour profiles of metabolites, glucose, and TAG with significant concentration differences between OW/OB and T2DM study groups. Mean \pm SEM concentrations of all amino acids $(n=9)$, biogenic amines $(n=3)$, acylcarnitines $(n=5)$, and the most significantly different phospholipids in the lean (red), OW/OB (blue), and T2DM (black) study groups are shown. Concentration (micromolar for metabolites and millimolar for glucose and TAG) is shown on the $y$ axis. The dark condition (10:30 PM to 6:30 AM, 0 lux) is illustrated on the graph as a gray-shaded area, and time of day (h) is displayed on the $x$ axis.

Six metabolites-proline, sarcosine, AC-C3, lysoPCa (C18:1 and C18:2), and PCaa (C36:5) - were affected by body mass, T2DM, and time of day (i.e., had robust daily rhythms in every study group and significant concentration differences between study groups and their controls). These were the only metabolites with significant differences in concentration between study groups that also exhibited significant 24 -h cosine rhythms in most of the participants in 
all study groups. Proline, sarcosine, and AC-C3 had significantly higher concentrations in the $\mathrm{OW} / \mathrm{OB}$ group compared with the lean group that were significantly higher still in the T2DM group compared with the OW/OB group. Lysophosphatidylcholines (C18:1 and C18:2) had significantly lower concentrations in the $\mathrm{OW} / \mathrm{OB}$ group compared with the lean group that were significantly lower still in the T2DM group compared with the OW/OB group. Diacylphosphatidylcholine (PCaa C36:5) did not follow this pattern, its concentrations being significantly lower in the OW/OB group compared with the lean group and significantly higher in the T2DM group compared with the OW/ OB group.

In addition to the effect of body mass and T2DM, more than one half of the metabolites [80 (61\%) of 130] exhibited a significant variation with time of day (ANOVA, FDR $<$ 0.05; Supplemental Table 3). No metabolites showed a significant time of day $\times$ group interaction, with the exception of lysoPCa 16:1 (lean vs. OW/OB group). We calculated the time point that best distinguished concentration differences between study groups. Metabolites that had significant concentration differences in both the OW/ OB group compared with lean controls and the T2DM group compared with $\mathrm{OW} / \mathrm{OB}$ controls-comprising one third of the metabolites $[n=40(31 \%)$ of 130$]$ - were used to determine the optimal sampling time. Concentration differences for these metabolites at each time point across $24 \mathrm{~h}$ are plotted as heatmaps (Fig. 6) and show the optimal time of day for sampling to maximize concentration differences between groups. For each metabolite, the time point at which its concentration differed the most between study groups is included in Supplemental Table 3. We also calculated the time point at which most metabolites within each class had the maximum concentration difference between study groups. The polar plot of these data that compares the lean and OW/OB groups (Supplemental Fig. $1 A$ ) illustrates that $30 \%$ of the amino acids and biogenic amines classes (combined) had their maximum differences in concentration at 1:00 AM. In contrast, 30\% of acylcarnitines had their maximum differences at 1:00 PM and $45 \%$ of the phospholipids had their maximum differences at 5:00 AM. Comparing the OW/OB and T2DM groups (Supplemental Fig. 1B), 20\% of the amino acids and biogenic amines (combined) had their maximum differences in concentration at 1:00 and 7:00 AM. In contrast, $40 \%$ of acylcarnitines had their maximum differences at 23:00 h, and $30 \%$ of phospholipids had their maximum differences at 5:00 PM, 10 and $12 \mathrm{~h}$ different from the lean and OW/OB groups, respectively. The optimum time of day to detect differences between study groups thus differed according to metabolite class and the study groups being compared.

\section{DISCUSSION}

This study, to our knowledge, is the first to independently map the effects of increased body mass and the combined effects of obesity and T2DM on 24-h plasma metabolite rhythms in men. Rhythmic metabolites were observed in all study groups (lean, $n=35$; OW /OB, $n=39$; T2DM, $n=20), 84 \%$ of which peaked during lights on. Metabolites that demonstrated significant daily rhythms are involved in the biochemical pathways that underlie T2DM and have been proposed as biomarkers (e.g., branched chain amino acids, short chain acylcarnitines, $\alpha$-AAA) (21-25). The robust time-of-day variation that was observed in the metabolite profiles highlights that time of day should be noted and controlled for clinical sampling, biomarker discovery, and interpretation of T2DM metabolomics studies.

Metabolites that exhibit significant cosine rhythms in more than 1 study group $(n=30)$ peaked at approximately the same time of day, thus suggesting that the timing of metabolite rhythms is not significantly affected by increased body mass or T2DM. This lack of change in the timing of metabolite rhythms is in agreement with our earlier work that showed no change in the timing of the plasma melatonin and leptin rhythms in these study groups (17). Similarly, there was no significant difference in the amplitude of the rhythmic metabolites between study groups. This finding contrasts with reports of attenuated gene expression circadian rhythmicity in adipose tissue in mouse models of obesity and T2DM (26) and in leukocytes of patients with T2DM (27). The disparity between the attenuated rhythms observed in gene expression and the retention of plasma metabolite rhythms in obesity and T2DM likely reflects the homeostatic mechanisms and multiple regulatory pathways that are present in the circulation. The comparable robust metabolite rhythms that were observed in both the $\mathrm{OW} / \mathrm{OB}$ and T2DM groups indicate that no general rule of reduced amplitude from lean to obese to T2DM can be made.

Daily rhythms in plasma metabolites have previously been characterized in lean, young male study participants by using the same targeted LC/MS-based metabolomics approach (12). Of the 171 metabolites measured in that study, 109 (64\%) exhibited a diurnal rhythm compared with only $27 \%$ rhythmic metabolites in the lean group in the present study. Although the participants in both studies had similar sleep-wake times, participants in the study by Davies et al. (12) had regular meals-breakfast, lunch, dinner, evening snack-rather than the hourly isocaloric meals in the present study, which resulted in set, imposed postprandial changes in circulating metabolites. In the present study, choosing to administer hourly isocaloric meals during the light period provided the opportunity to test postabsorptive metabolism while removing the imposed effects of large meals on metabolite profiles. Despite these differences in study protocols, significant 24-h cosine rhythms were observed in some of the same metabolites of the study by Davies et al. (12) and the lean group of the present study, namely, isoleucine, proline, valine, kynurenine, symmetrical dimethylarginine, sarcosine, $\alpha$-AAA, acetylcarnitine, butyrylcarnitine, hexadecanoylcarnitine, AC-C18:1, lysoPCa C18:2, PCaa C40:2, PCaa C42:2, and SM C20:2. Both glucose and TAG levels peaked in the afternoon, which is in agreement with earlier studies under similar, regular, small meal feeding conditions (28), and are most likely a result of insulin resistance in the afternoon. Whereas nutritional status undoubtedly affects circulating metabolite levels, there is also evidence to suggest that some of these metabolites are under circadian clock control—rhythmic expression being present in 


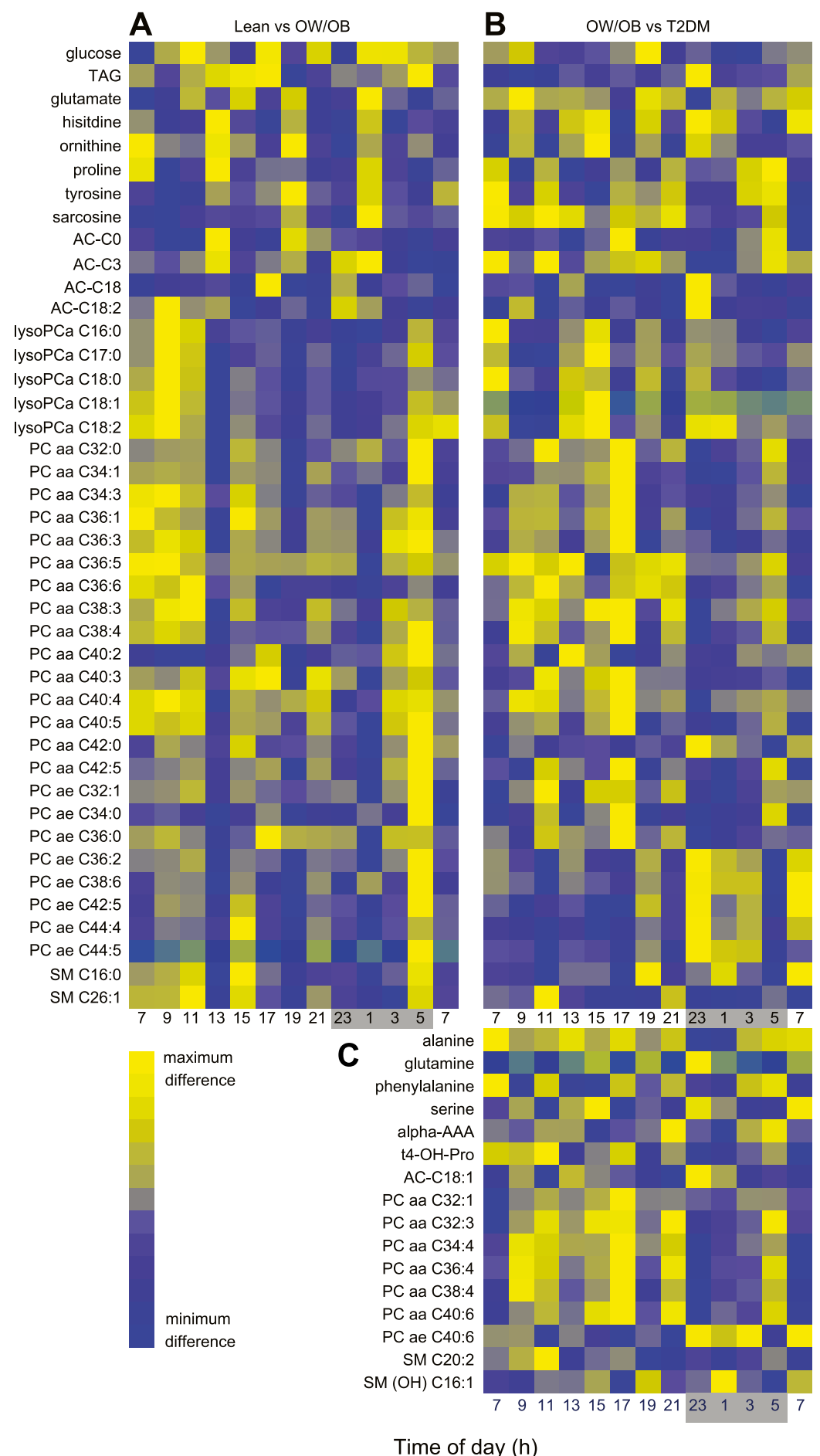

Figure 6. Heatmaps showing optimal time of day for maximizing sampling differences between study groups. A) Time points of maximum (yellow) or minimum (blue) concentration difference between $\mathrm{OW} / \mathrm{OB}$ and lean groups across $24 \mathrm{~h} . B, C$ ) Time points of maximum (yellow) or minimum (blue) concentration difference between T2DM and OW/OB groups across $24 \mathrm{~h}$. The dark condition (10:30 PM to $6: 30 \mathrm{AM}, 0 \mathrm{lux})$ is illustrated on the $x$ axis as a grayshaded area. laboratory constant routine conditions that are optimized to reveal endogenous circadian rhythms $(8,9,11,28)$.

Previous studies that have compared the metabolic profiles of individuals who went on to develop T2DM with those that did not have helped to uncover novel T2DM biomarkers (21-25). Although not all studies identified the same metabolites, a T2DM metabolic phenotype may include higher concentrations of alanine, glutamate, phenylalanine, tyrosine, $\alpha$-AAA, t4-OH-Pro, and branched-chain amino acids (leucine, isoleucine, and valine), as well as lower concentrations of glycine, serine, and glutamine. Of note, in the present study, significantly higher alanine, phenylalanine, $\alpha-\mathrm{AAA}$, and $\mathrm{t} 4-\mathrm{OH}-\mathrm{Pro}$ levels, as well as lower glutamine and serine levels were only observed in T2DM compared with the OW/OB group and, therefore, may arise independently of increased body mass. Individuals with $\alpha$-AAA concentrations in the top quartile show the strongest association with diabetes risk, high $\alpha$-AAA levels being associated with insulin resistance and impaired $\beta$-cell function (24). Elevated $\alpha$-AAA levels are associated with low concentrations of glutathione, likely as a result of inflammation 
and oxidative stress, which are common comorbidities of T2DM (21) that are consistent with the raised $\alpha$-AAA levels in our T2DM group. Elevated daytime $\alpha$-AAA concentrations also resulted in loss of the $\alpha$-AAA rhythm in the T2DM group. Alterations in the shape of the 24-h profiles, which resulted in loss of the cosine rhythm, in some key amino acids that are involved in T2DMnamely, alanine, glycine, isoleucine, tyrosine, and valine-suggest defective 24 -h processing in T2DM that requires additional study.

Metabolites that demonstrated significant changes in $\mathrm{OW} / \mathrm{OB}$ compared to the lean group, and in T2DM compared to the OW/OB group could be on a continuum from lean to obesity to T2DM. For example, in the current study, significantly higher short-chain acylcarnitine (AC$\mathrm{C} 0$ and $\mathrm{AC}-\mathrm{C} 3$ ) levels were observed in the $\mathrm{OW} / \mathrm{OB}$ and T2DM groups. Similar findings have also been reported previously (29), with higher acylcarnitine concentrations possibly resulting from excessive nonesterified fatty acid (NEFA) availability, reduced NEFA oxidation, or both, as malonyl-CoA inhibition of carnitine palmitoyltransferase 1 slows the transfer of NEFA into the mitochondria (29).

T2DM had no consistent effect on phospholipids, with both higher $(n=22)$ and lower levels $(n=13)$ in the T2DM group compared with the OW/OB control group. The effect of T2DM on specific phospholipid species varies between metabolomics studies, so much so that a metaanalysis could not be performed on the lipid class as a result of high interstudy inconsistency (25). Plasma lysoPCs were negatively associated with BMI and plasma insulin concentrations, but had no relationship with glucose and the homeostatic model assessment-insulin resistance, which suggests that adiposity underlies the observed changes rather than insulin resistance or T2DM (5); however, in the current study, lysoPC responses seem to be more consistent-lysoPCs with significant group differences had lower levels in the OW/OB group that were lower still in T2DM, which suggests that they are affected by both weight and T2DM. Rhythmic lysoPCs also displayed robust rhythms, with the exception of C26:1, that all peaked in the evening.

We performed quantitative enrichment analysis; however, no pathways were identified as being significantly over-represented after FDR correction. Several metabolites that exhibited robust daily rhythms in the majority of participants in all study groups and that also showed a significant progressive change in concentration from lean to $\mathrm{OW} / \mathrm{OB}$ to T2DM groups were identified. These metabolites, namely, proline, sarcosine, AC-C3, and lysoPCa (C18:1 and C18:2), may play a role or act as biomarkers in the progression from a healthy weight to obese with T2DM. These metabolites are worthy of future study to identify underlying mechanisms and to increase our understanding of the interaction between T2DM and the circadian timing system.

The time series sampling also afforded the opportunity to examine the optimum sampling time at which to best distinguish differences between study groups. For each metabolite, an optimum time of collection was determined to provide better discriminating biomarkers. The rhythmic nature of most metabolites meant that the best sampling time differed depending on the metabolite, metabolite class, and the study groups being compared. We observed some large differences (10-12h) between study groups, for example, acylcarnitines showed maximal difference at 1:00 (lean vs. OW/OB) and at 11:00 PM (OW/OB vs. T2DM), and phospholipids showed maximal difference at 5:00 AM (lean vs. OW/OB) and at 5:00 PM (OW/OB vs. T2DM). In contrast, the optimum sampling time for amino acids and biogenic amines was 01:00 h, irrespective of the study groups being compared. Despite being unable to generate any general rules for the optimum sampling of metabolites, it is interesting to note that none of the metabolite classes exhibited its maximum concentration differences between 7:00 AM and 11:00 PM, when samples are typically drawn for diagnostic and research purposes. These findings highlight the importance of controlling the time of day and highlight the value of metabolic profiling across $24 \mathrm{~h}$.

\section{CONCLUSIONS}

To our knowledge, this is the first time a 24-h time series of plasma metabolites has been simultaneously assessed in T2DM compared with an age- and weight-matched control group during a controlled daily routine. It is clear that many aspects of the altered metabolome in T2DM exhibit 24-h diurnal variation, which has previously been overlooked in studies that used only single samples that were taken at a poorly defined time point. Our results reveal that the effect of body mass and T2DM on the rhythmic metabolome vary in a metabolite-specific manner. Furthermore, our findings highlight how the time of day regulates metabolite differences between lean, $\mathrm{OW} / \mathrm{OB}$, and T2DM phenotypes. These data thus increase our understanding of the interactions between circadian and metabolic physiology and will inform the experimental design of future studies.

\section{ACKNOWLEDGMENTS}

The authors thank Dr. Daniella T. Otway for recruitment of participants and for contributing to data collection, and Dr. Denise Robertson for input into study design and sample collection (both from the Faculty of Health and Medical Sciences, University of Surrey). The assistance of Namrata Chowdhury and the staff of the Surrey Clinical Research Centre and the Metabolomics Core Facility at the University of Surrey is acknowledged. The authors also thank Dr. Anne Skeldon (Department of Mathematics, University of Surrey) and Dr. Jeroen Pennings (National Institute for Public Health and the Environment, Bilthoven, The Netherlands, for assistance and writing the analysis scripts for MatLab and R, respectively. This work was funded by Diabetes United Kingdom (Grant 08/0003607), EU FP7-HEALTH-2011 EuRhythDia (Grant 278397), the Biotechnology and Biological Sciences Research Council (Grants BB/D526853/1 and BB/I019405/1), and Stockgrand Ltd. (Guilford, United Kingdom). The authors declare no conflicts of interest.

\section{AUTHOR CONTRIBUTIONS}

C. M. Isherwood wrote the manuscript and analyzed the data; D. R. Van der Veen revised the manuscript and 
contributed to the interpretation of the data; J. D. Johnston revised the manuscript, designed the study, and contributed to the interpretation of the data; and D. J. Skene wrote the manuscript, designed the study and contributed to the interpretation of the data.

\section{REFERENCES}

1. Mokdad, A. H., Ford, E. S., Bowman, B. A., Dietz, W. H., Vinicor, F., Bales, V. S., and Marks, J. S. (2003) Prevalence of obesity, diabetes, and obesity-related health risk factors, 2001. JAMA 289, 76-79

2. Newgard, C. B., An, J., Bain, J. R., Muehlbauer, M. J., Stevens, R. D., Lien, L. F., Haqq, A. M., Shah, S. H., Arlotto, M., Slentz, C. A., Rochon, J., Gallup, D., Ilkayeva, O., Wenner, B. R., Yancy, W. S., Jr., Eisenson, H., Musante, G., Surwit, R. S., Millington, D. S., Butler, M. D., and Svetkey, L. P. (2009) A branched-chain amino acid-related metabolic signature that differentiates obese and lean humans and contributes to insulin resistance. Cell Metab. 9, 311-326

3. Suhre, K., Meisinger, C., Döring, A., Altmaier, E., Belcredi, P., Gieger, C., Chang, D., Milburn, M. V., Gall, W. E., Weinberger, K. M., Mewes, H. W., Hrabé de Angelis, M., Wichmann, H. E., Kronenberg, F., Adamski, J., and Illig, T. (2010) Metabolic footprint of diabetes: a multiplatform metabolomics study in an epidemiological setting. PLoS One 5, e13953

4. Mihalik, S. J., Michaliszyn, S. F., de las Heras, J., Bacha, F., Lee, S., Chace, D. H., DeJesus, V. R., Vockley, J., and Arslanian, S. A. (2012) Metabolomic profiling of fatty acid and amino acid metabolism in youth with obesity and type 2 diabetes: evidence for enhanced mitochondrial oxidation. Diabetes Care 35, 605-611

5. Barber, M. N., Risis, S., Yang, C., Meikle, P. J., Staples, M., Febbraio, M. A., and Bruce, C. R. (2012) Plasma lysophosphatidylcholine levels are reduced in obesity and type 2 diabetes. PLoS One 7, e41456

6. Minami, Y., Kasukawa, T., Kakazu, Y., Iigo, M., Sugimoto, M., Ikeda, S., Yasui, A., van der Horst, G. T., Soga, T., and Ueda, H. R. (2009) Measurement of internal body time by blood metabolomics. Proc. Natl. Acad. Sci. USA 106, 9890-9895

7. Ang, J. E., Revell, V., Mann, A., Mäntele, S., Otway, D. T., Johnston, J. D., Thumser, A. E., Skene, D. J., and Raynaud, F. (2012) Identification of human plasma metabolites exhibiting time-of-day variation using an untargeted liquid chromatography-mass spectrometry metabolomic approach. Chronobiol. Int. 29, 868-881

8. Dallmann, R., Viola, A. U., Tarokh, L., Cajochen, C., and Brown, S. A. (2012) The human circadian metabolome. Proc. Natl. Acad. Sci. USA 109, 2625-2629

9. Kasukawa, T., Sugimoto, M., Hida, A., Minami, Y., Mori, M., Honma, S., Honma, K., Mishima, K., Soga, T., and Ueda, H. R. (2012) Human blood metabolite timetable indicates internal body time. Proc. Natl. Acad. Sci. USA 109, 15036-15041

10. Krug, S., Kastenmüller, G., Stückler, F., Rist, M.J., Skurk, T., Sailer, M., Raffler, J., Römisch-Margl, W., Adamski, J., Prehn, C., Frank, T., Engel, K. H., Hofmann, T., Luy, B., Zimmermann, R., Moritz, F., Schmitt-Kopplin, P., Krumsiek, J., Kremer, W., Huber, F., Oeh, U., Theis, F. J., Szymczak, W., Hauner, H., Suhre, K., and Daniel, H. (2012) The dynamic range of the human metabolome revealed by challenges. FASEB J. 26, 2607-2619

11. Chua, E. C. P., Shui, G., Lee, I. T., Lau, P., Tan, L. C., Yeo, S. C., Lam, B. D., Bulchand, S., Summers, S. A., Puvanendran, K., Rozen, S. G., Wenk, M. R., and Gooley, J. J. (2013) Extensive diversity in circadian regulation of plasma lipids and evidence for different circadian metabolic phenotypes in humans. Proc. Natl. Acad. Sci. USA 110, 14468-14473

12. Davies, S. K., Ang, J. E., Revell, V. L., Holmes, B., Mann, A., Robertson, F. P., Cui, N., Middleton, B., Ackermann, K., Kayser, M., Thumser, A. E., Raynaud, F. I., and Skene, D. J. (2014) Effect of sleep deprivation on the human metabolome. Proc. Natl. Acad. Sci. USA 111, 10761-10766

13. Scheer, F. A. J. L., Hilton, M. F., Mantzoros, C. S., and Shea, S. A. (2009) Adverse metabolic and cardiovascular consequences of circadian misalignment. Proc. Natl. Acad. Sci. USA 106, 4453-4458

14. Potter, G. D., Skene, D. J., Arendt, J., Cade, J. E., Grant, P. J., and Hardie, L. J. (2016) Circadian rhythm and sleep disruption: causes, metabolic consequences, and countermeasures. Endocr. Rev. 37, 584-608

15. Johnston, J. D., Ordovás, J. M., Scheer, F. A., and Turek, F. W. (2016) Circadian rhythms, metabolism, and chrononutrition in rodents and humans. Adv. Nutr. 7, 399-406

16. Otway, D. T., Mäntele, S., Bretschneider, S., Wright, J., Trayhurn, P., Skene, D. J., Robertson, M. D., and Johnston, J. D. (2011) Rhythmic diurnal gene expression in human adipose tissue from individuals who are lean, overweight, and type 2 diabetic. Diabetes 60, 1577-1581

17. Mäntele, S., Otway, D. T., Middleton, B., Bretschneider, S., Wright, J., Robertson, M. D., Skene, D. J., and Johnston, J. D. (2012) Daily rhythms of plasma melatonin, but not plasma leptin or leptin mRNA, vary between lean, obese and type 2 diabetic men. PLoS One 7, e37123

18. Schofield, W. N. (1985) Predicting basal metabolic rate, new standards and review of previous work. Hum. Nutr. Clin. Nutr. 39 (Suppl 1), 5-41

19. Skene, D. J., Middleton, B., Fraser, C. K., Pennings, J. L., Kuchel, T. R., Rudiger, S. R., Bawden, C. S., and Morton, A. J. (2017) Metabolic profiling of presymptomatic Huntington's disease sheep reveals novel biomarkers. Sci. Rep. 7, 43030

20. Xia, J., Sinelnikov, I. V., Han, B., and Wishart, D. S. (2015) MetaboAnalyst 3.0-making metabolomics more meaningful. Nucleic Acids Res. 43, W251-W257

21. Wang, T. J., Larson, M. G., Vasan, R. S., Cheng, S., Rhee, E. P., McCabe, E., Lewis, G. D., Fox, C. S., Jacques, P. F., Fernandez, C., O'Donnell, C. J., Carr, S. A., Mootha, V. K., Florez, J. C., Souza, A., Melander, O., Clish, C. B., and Gerszten, R. E. (2011) Metabolite profiles and the risk of developing diabetes. Nat. Med. 17, 448-453

22. Wang-Sattler, R., Yu, Z., Herder, C., Messias, A. C., Floegel, A., He, Y., Heim, K., Campillos, M., Holzapfel, C., Thorand, B., Grallert, H., Xu, T., Bader, E., Huth, C., Mittelstrass, K., Döring, A., Meisinger, C., Gieger, C., Prehn, C., Roemisch-Margl, W., Carstensen, M., Xie, L., Yamanaka-Okumura, H., Xing, G., Ceglarek, U., Thiery, J., Giani, G., Lickert, H., Lin, X., Li, Y., Boeing, H., Joost, H. G., de Angelis, M. H., Rathmann, W., Suhre, K., Prokisch, H., Peters, A., Meitinger, T., Roden, M., Wichmann, H. E., Pischon, T., Adamski, J., and Illig, T. (2012) Novel biomarkers for pre-diabetes identified by metabolomics. Mol. Syst. Biol. 8, 615

23. Floegel, A., Stefan, N., Yu, Z., Mühlenbruch, K., Drogan, D., Joost, H. G., Fritsche, A., Häring, H. U., Hrabě de Angelis, M., Peters, A., Roden, M., Prehn, C., Wang-Sattler, R., Illig, T., Schulze, M. B., Adamski, J., Boeing, H., and Pischon, T. (2013) Identification of serum metabolites associated with risk of type 2 diabetes using a targeted metabolomic approach. Diabetes 62, 639-648

24. Wang, T. J., Ngo, D., Psychogios, N., Dejam, A., Larson, M. G., Vasan, R. S., Ghorbani, A., O'Sullivan, J., Cheng, S., Rhee, E. P., Sinha, S., McCabe, E., Fox, C. S., O’Donnell, C. J., Ho, J. E., Florez, J. C., Magnusson, M., Pierce, K. A., Souza, A. L., Yu, Y., Carter, C., Light, P. E., Melander, O., Clish, C. B., and Gerszten, R. E. (2013) 2Aminoadipic acid is a biomarker for diabetes risk. J. Clin. Invest. 123, 4309-4317

25. Guasch-Ferré, M., Hruby, A., Toledo, E., Clish, C. B., Martínez-González, M. A., Salas-Salvadó, J., and Hu, F. B. (2016) Metabolomics in prediabetes and diabetes: a systematic review and meta-analysis. Diabetes Care 39, 833-846

26. Ando, H., Yanagihara, H., Hayashi, Y., Obi, Y., Tsuruoka, S., Takamura, T., Kaneko, S., and Fujimura, A. (2005) Rhythmic messenger ribonucleic acid expression of clock genes and adipocytokines in mouse visceral adipose tissue. Endocrinology 146, 5631-5636

27. Ando, H., Takamura, T., Matsuzawa-Nagata, N., Shima, K. R., Eto, T., Misu, H., Shiramoto, M., Tsuru, T., Irie, S., Fujimura, A., and Kaneko, S. (2009) Clock gene expression in peripheral leucocytes of patients with type 2 diabetes. Diabetologia 52, 329-335

28. Van Cauter, E., Polonsky, K. S., and Scheen, A. J. (1997) Roles of circadian rhythmicity and sleep in human glucose regulation. Endocr. Rev. 18, 716-738

29. Mihalik, S.J., Goodpaster, B. H., Kelley, D. E., Chace, D. H., Vockley, J., Toledo, F. G., and DeLany, J. P. (2010) Increased levels of plasma acylcarnitines in obesity and type 2 diabetes and identification of a marker of glucolipotoxicity. Obesity (Silver Spring) 18, 1695-1700 